\title{
Comment to: Pre-peritoneal local anaesthetic does not reduce post-operative pain in laparoscopic total extra-peritoneal inguinal hernia repair: double-blinded randomized controlled trial. Kulasegaran S, Rohan M, Pearless L, Hulme-Moir M
}

\author{
Lucas J. Castro-Alves ${ }^{1} \cdot$ Mark C. Kendall $^{1}$
}

Received: 7 January 2018 / Accepted: 12 January 2018 / Published online: 22 January 2018

(c) Springer-Verlag France SAS, part of Springer Nature 2018

To the Editor,

We read with great interest the article of Kulasegaranet al. [1] in a recent issue of the journal. The authors evaluated one hundred patients undergoing laparoscopic total preperitoneal hernia repair and concluded that pre-peritoneal local anaesthetic instillation does not offer any advantage in pain scores at 4 and $24 \mathrm{~h}$ after surgery. The authors should be congratulated for performing a well-designed study in an important topic (e.g. acute pain) in patients undergoing hernia repair [2,3]. Moreover, local anesthetic instillation has become the current emphasis on many studies evaluating acute pain $[4,5]$.

Although the study of Kulasegaran et al. was well conducted, there are some concerns regarding the trial that needs to be clarified to determine the validity of the results. First, it is not clear if intraoperative analgesic administration was standardized using a specific criterion as this can alter the primary outcome [6]. Secondly, as fentanyl was used to treat postoperative pain, it is expected that patients were treated to keep low postoperative pain. One could argue that this is the reason for the lack of analgesic benefit demonstrated by the study. Lastly, the authors performed a presurgical versus post-surgical analysis in Table 2 . It would be important to demonstrate a simple pain analysis by study groups since the $P$ value for the pre- and post-analyses was almost statistically significant $(P=0.06)$.

This comment refers to the article available at https://doi. org/10.1007/s10029-017-1672-1.

Mark C. Kendall

mark.kendall@lifespan.org

Department of Anesthesiology, Rhode Island Hospital, Warren Alpert Medical School of Brown University, 593 Eddy Street, Providence, RI 02903, USA
We would welcome some comments to address the aforementioned issues. This would help to further substantiate the findings of this important study.

\section{Compliance with ethical standards}

Conflict of interest No conflicts of interest to report.

Ethical approval This comment did not need approval from an ethic committee.

Statement on human and animal rights This comment does not contain any studies with participants or animals.

Informed consent Informed consent was not required for this comment.

\section{References}

1. Kulasegaran S, Rohan M, Pearless L, Hulme-Moir M (2017) Preperitoneal local anaesthetic does not reduce post-operative pain in laparoscopic total extra-peritoneal inguinal hernia repair: doubleblinded randomized controlled trial. Hernia 21:879-885

2. Pere P, Harju J, Kairaluoma P, Remes V, Turunen P, Rosenberg PH (2016) Randomized comparison of the feasibility of three anesthetic techniques for day-case open inguinal hernia repair. J Clin Anesth 34:166-175

3. Wright R, Born DE, D'Souza N, Hurd L, Gill R, Wright D (2017) Pain and compression neuropathy in primary inguinal hernia. Hernia 21:715-722

4. Cip J, Erb-Linzmeier H, Stadlbauer P, Bach C, Martin A, Germann R (2016) Continuous intra-articular local anesthetic drug instillation versus discontinuous sciatic nerve block after total knee arthroplasty. J Clin Anesth 35:543-550

5. Gurusamy KS, Nagendran M, Guerrini GP, Toon CD, Zinnuroglu M, Davidson BR (2014) Intraperitoneal local anaesthetic instillation versus no intraperitoneal local anaesthetic instillation for laparoscopic cholecystectomy. Cochrane Database Syst Rev 3:007337

6. Koo CH, Cho YJ, Hong DM, Jeon Y, Kim TK (2016) Influence of high-dose intraoperative remifentanil with intravenous ibuprofen on postoperative morphine consumption in patients undergoing pancreaticoduodenectomy: a randomized trial. J Clin Anesth 35:47-53 